\title{
Perspective of auto parts enterprises in whole life cycle cost management research
}

\author{
SHAO Wen-wu, XIAO Yin-tao ${ }^{1, a}$, \\ (School of Shenyang Aerospace University, Shenyang, 110000) \\ a,2419937036@qq.com
}

Keywords: full life cycle; auto parts; cost management

\begin{abstract}
In order to solve the problem of cost management faced by enterprises, this paper uses the method of total life cycle cost management to analyze and manage the cost of each stage of production process of the enterprise, and puts forward the idea of using dynamic programming to effectively manage the cost of the whole process of product production, As an effective way for enterprises to allocate costs, optimize management decisions and improve the profitability of enterprises. The research shows that cost management of the whole life cycle helps to strengthen the operation and management of enterprises. It is a kind of full participation, including the cost management of the whole process of product production.
\end{abstract}

\section{Introduction}

In the changing process of the competitive environment of enterprises, the drawbacks of the traditional cost management mode are constantly appearing. First, the traditional cost management mainly focuses on the cost control in the matter and the manufacturing cost as the key control point. However, in the production process of the enterprise product, The cost management requirements have been extended to the product development stage and product after-sales service phase, which requires the formation of a new business model - full life-cycle cost management. Second, the traditional cost management focuses on the goal of economic benefits, the pursuit of local cost optimization, ignoring the various stages of product production linkages, in the production process, the cost of information collection and consolidation did not establish a unified and standardized standards In order to obtain accurate cost information, enterprises need to establish a cost integration model based on the whole life cycle, effectively classify and organize the collected cost information, and become the basis for enterprises to make management and decision-making. Under this background, under the guidance of the whole life-cycle cost management theory, this paper applies the dynamic programming theory to rationally allocate the cost of the product in all stages of the life cycle and make the efficient use of resources. On the basis of ensuring the completion of production tasks, Get higher profits, so that enterprises in the incentive competition better development.

\section{Theories of Life Cycle Cost Management}

\subsection{Full life-cycle cost management goals}

The goal of strategic management is to promote the better development of enterprises and improve their competitive advantages. Cost-based life-cycle-based management also helps enterprises to achieve this goal. The whole life-cycle cost management objectives include the following aspects: 1 . The basic functions of the whole life-cycle and cost management in order to better the development of enterprises to maintain their competitive edge. 2 . The cost of life-cycle management of the enterprise's resources to make a reasonable allocation, increase corporate profits. 3. In order to realize the sustainable development of enterprises, the methods taken at different stages of the life cycle will be different, but the goal of cost management is to realize the overall optimization. 


\subsection{Full life cycle cost management features}

The whole life-cycle theory changes the concept of cost management from a static cost management concept confined to a certain stage to the concept of system, association and dynamic cost management. Cost management from the perspective of life cycle has the characteristics of general cost management, but also has the characteristics of integrity, foresight and relevance:

(1) Integrity

Total life cycle cost management is based on the production process of the product, including the cost management of the $\mathrm{R} \& \mathrm{D}$ phase, procurement phase, manufacturing phase, sales phase and after-sales service phase of the enterprise for the production of products. Compared with the traditional cost management, Cost management of the life cycle concerned about the cost of products in all stages of consumption, more accurate and efficient accounting for the cost of incentives, reflecting the cost management requirements.

(2) Prospective

The whole life cycle cost management is the enterprise in the product R \& D and design phase, through the analysis of customers and markets to understand the customer demand for products, features and price acceptance, and then product features, production processes R \& D design. Through the cost management in the research and development stage, the guidance function of market orientation on cost management was expanded.

(3) Relevance

Based on life cycle cost management, we consider the relationship among all stages and analyze the relationship between costs and benefits in each stage. The cost will be maximized based on the completion of production tasks. Therefore, the whole life-cycle cost management not only includes the various stages in the scope, but more importantly, it shows the connection between the stages, helps the rational allocation of resources, and reflects the synergy of all activities in the whole life cycle.

2.3 Full life-cycle cost management

(1) research and development stage

The R \& D stage is mandatory for the costs incurred in other production stages. Cost information in the $\mathrm{R} \& \mathrm{D}$ phase mainly includes the cost information of process design and material design. The cost information of the process design mainly includes the production process of the product, the design of the machinery and equipment and the labor demand, etc. The cost information of the material design includes the performance of the purchased material, the index, the demand, and the design of the specification. The cost information in the $\mathrm{R} \& \mathrm{D}$ stage is mainly the reference and guideline for the cost of the subsequent stages.

(2) Procurement phase and manufacturing phase

Procurement stage and manufacturing stage are the main stages of the cost of consumption, the traditional cost management as the focus of this study. The two stages of mining and manufacturing activities are based on the R \& D stage design, cost management is more passive, the actual cost of production will change, but it is difficult to directly find the reasons for the cost difference. Cost information in the procurement phase The main purchasing material performance, indicators, specifications and prices and other information. The cost information of the manufacturing stage includes the name of material consumed in the material cost, the procurement cost of the unit, the actual consumption amount, the man-hours in the processing cost, the working hours of the machinery and equipment and other information.

(3) sales stage and after-sales service stage

At the end of the sales and post-sales phases, the production costs of the products are basically completed. The cost of production and the customer information are mainly fed back so that the design and production in the research and development phase, the procurement phase and the manufacturing phase more meet the market demand. These two stages mainly include the sale of products, product maintenance, maintenance and other information. 


\section{Dynamic life cycle cost planning}

Dynamic cost planning is based on the product's production process. Through the research on the various stages of the product, the limited resources are rationally allocated to each stage of the product's production process. In the production process of the enterprise's products, the factors that constantly change in various stages of the product cost are considered, Real-time control of the cost of occurrence, to better achieve business objectives, to create more profits.

(1) establish a cost database

The cost database is the cost data of all stages of an enterprise's life cycle. After processing and processing, various cost indices are formed to support the product development. The enterprise can use these cost information as the basis for establishing reasonable cost Budget model for project decision making. In the production process, cost data provides the cost of resources in various stages of the completion of various tasks, the cost of each dimension contrast, you can quickly find the cost management process problems, the product is completed, based on historical production data , You can create a cost database based on standard costs.

(2) set the target cost

In the process of cost dynamic planning, all the tasks are based on the target cost of development, so that the target cost can participate in the complete dynamic production process, so that employees can understand the occurrence and change of costs in time and set the target cost mainly following the following principles : a.The principle of enforceability: The target cost is not only the need of enterprise cost management, but also the practical tool of cost management. b. The principle of stage: the completion of the target cost management should be implemented in different stages of different requirements and accuracy of the cost of preparation.c.Principle of Cost and Benefit: Since dynamic cost planning needs to compare the costs and benefits of various tasks, it is necessary to consider the value creation corresponding to cost consumption in formulating a detailed target cost.

(3) cost allocation

The target cost of the whole life cycle is determined, the total target cost is allocated to each stage, the phases are divided into the activities for completing the task, the dynamic programming of resources is used to allocate certain resources for each task in the process of the cost allocation, Activities of resource allocation to determine the various stages of resource consumption information, according to the unit price of these resources, you can determine the tasks, the cost of each stage.

\section{Dynamic life cycle cost planning}

In the process of dynamic cost management, the dynamic planning needs to separate the current period from the future one, but the current cost and the future benefit should be considered in the cost optimization method. Therefore, the selection of each phase should be based on the global optimization consider. The key of cost dynamic programming lies in correctly writing the basic recurrence relation and the proper basic equation. This requires dividing the process of the problem into several interrelated stages, selecting the state variables and decision variables reasonably, and defining the optimal function, So that a large problem can be solved into a group of similar sub-problems and then solved one by one. In each sub-problem, the optimization results of the sub-problems before it are used, and the final sub-problems The optimal solution obtained is the optimal solution to the entire problem. In general, the basic steps to establish a dynamic programming model can be summarized as the following five steps:

(1) Division stage

The first step of dynamic programming to solve multi-stage decision-making problem is to divide the stage. After determining the characteristics of each stage,In accordance with the order of time, the production process is divided into several interlinked stages.

(2) Select the state variables 
Correct choice of state variables, $\lambda_{k}$ said the k-phase of a state, it is necessary to accurately describe the process of evolution, but also to meet the non-aftermath, and the state variables of various stages of the value can be determined.

(3) Determine the decision variables and allow decision sets

The key variables of the problem to be solved are usually chosen as decision variables $\chi_{k}$, $\chi_{k}\left(\lambda_{k}\right)$ represents the decision variables in the kth state, The set of allowed decisions is made up of the range limited by the decision variables, $X_{k}\left(\lambda_{k}\right)$ represents the kth stage from the set of decision-making. The whole process of the strategy $\chi_{k}\left(\lambda_{k}\right)$ is composed

of decision-making variables of each stage sequence of decision-making functions, $p_{1}\left(\lambda_{1}\right)=\left\{\chi_{1}\left(\lambda_{1}\right), \chi_{2}\left(\lambda_{2}\right), \ldots, \chi_{n}\left(\lambda_{n}\right)\right\}$

(4) determine the state transfer equation

Dynamic programming optimization process is to solve all the stages of the decision-making, the state transfer equation describes the state of the transfer rule, if the k-stage state variables, $\mathrm{k}+1$ state variables will be followed.

(5) to determine the index function and the optimal index function

Index function refers to the k-phase consumption of certain resources generated after the income, the optimal indicator function is to consume a certain amount of resources to obtain the best value for money. Indicator functions written in constructing the basic equations of dynamic programming $V_{k}$

$$
V_{k}\left[\lambda_{k}, p_{k}\left(\lambda_{k}\right)\right]=V_{k}\left(\lambda_{k}, \chi_{k}\right)+V_{k+1}\left[\lambda_{k+1}, p_{k+1}\left(\lambda_{k+1}\right)\right]
$$

The sub-strategy $p_{k}\left(\lambda_{k}\right)$ is composed of $\chi_{k}\left(\lambda_{k}\right)$ and $p_{k+1}\left(\lambda_{k+1}\right)$

$$
p_{k}\left(\lambda_{k}\right)=\left\{\chi_{k}\left(\lambda_{k}\right), p_{k+1}\left(\lambda_{k+1}\right)\right\}(3)
$$

If we use $p_{k}{ }^{*}\left(\lambda_{k}\right)$ optimal sub-strategy which means that the initial state is the sub-strategy of all sub-strategies of the latter sub-processes, The optimal function can be expressed as:

$$
g_{k}\left(\lambda_{k}\right)=V_{k}\left[\lambda_{k}, p_{k}{ }^{*}\left(\lambda_{k}\right)\right]=\operatorname{opt}_{p_{k}}\left[\lambda_{k}, p_{k}\left(\lambda_{k}\right)\right]_{(4)}
$$

Then The optimal function can be expressed as:

$$
g_{k}\left(\lambda_{k}\right)=\underset{\chi_{k} \in X_{k}\left(\lambda_{k}\right)}{\operatorname{opt}}\left[V_{k}\left(\lambda_{k}, \chi_{k}\right)+g_{k+1}\left(\lambda_{k+1}\right)\right],(k=1.2 . \cdots, n-1)
$$

\section{Conclusion}

Cost management throughout the life cycle helps to strengthen the management of the enterprise. It is a full participation, including the cost management of the whole process of product production. According to the idea of total life cycle cost, enterprises should establish a systematic management thinking and further extend the scope of cost management to the research and development stage, procurement stage, sales and after-sales service stage. On the cost management approach, pay attention to the mutual In the concept of cost management, the cost management of each stage of the product's life cycle closely matches the development goal of the enterprise, combining the short-term benefits with the long-term benefits.

\section{References}

[1] Janz D .Design to life cycleby value-oriented life cycle costing [C].Advances in Life Cycle Engineering for Sustainable Manufacturing Busi-nesses.2010: 461-466

[2]Marketa Spickova,Renata Myskova. Costs Efficiency Evaluation using Life Cycle Costing as Strategic Method[J]. Procedia Economics and Finance,2015,34. 
[3]Marcus Bengtsson, Martin Kurdve. Machining Equipment Life Cycle Costing Model with Dynamic Maintenance Cost[J]. Procedia CIRP,2016,48. 